\title{
Comparison of triple dose versus standard dose gadolinium-DTPA for detection of MRI enhancing lesions in patients with primary progressive multiple sclerosis
}

Massimo Filippi, Adriana Campi, Vittorio Martinelli, Bruno Colombo, Tarek Yousry, Nicola Canal, Giuseppe Scotti, Giancarlo Comi

\begin{abstract}
This study was performed to evaluate whether a triple dose of gadoliniumDTPA (Gd-DTPA) increases the sensitivity of brain MRI for detecting enhancing lesions in patients with primary progressive multiple sclerosis (PPMS). T1 weighted brain MRI was obtained for 10 patients with PPMS in two sessions. In the first session, one scan was obtained five to seven minutes after the injection of $0.1 \mathrm{mmol} / \mathrm{kg}$ Gd-DTPA (standard dose). In the second session, six to 24 hours later, one scan before and two scans five to seven minutes and one hour after the injection of $0.3 \mathrm{mmol} / \mathrm{kg}$ Gd-DTPA (triple dose) were obtained. Four enhancing lesions were detected in two patients when the standard dose of Gd-DTPA was used. The numbers of enhancing lesions increased to 13 and the numbers of patients with such lesions to five when the triple dose of Gd-DTPA was used and to 14 and six in the one hour delayed scans. The mean contrast ratio for enhancing lesions detected with the triple dose of Gd-DTPA was higher than those for lesions present in both the standard dose $(P<0.0009)$ and the one hour delayed scans $(P=0 \cdot 04)$. These data indicate that with a triple dose of Gd-DTPA many more enhancing lesions can be detected in patients with PPMS. This is important both for planning clinical trials and for detecting the presence of inflammation in vivo in the lesions of such patients.
\end{abstract}

$(\Im$ Neurol Neurosurg Psychiatry 1995;59:540-544)

Keywords: primary progressive multiple sclerosis; magnetic resonance imaging; gadolinium-DTPA.

Several recent studies have shown that gadolinium (Gd)-enhanced MRI detects new pathological activity five to 10 times more often than clinical relapse does in patients with relapsing-remitting or secondary progressive multiple sclerosis ${ }^{1-6}$ and, therefore, it is at present used both for screening the effects of new putative treatments and as a supplementary marker of disease activity in large scale phase III clinical trials. ${ }^{7}$
Patients with primary progressive multiple sclerosis (PPMS) typically have few brain MRI abnormalities on T2 weighted scans, and they rarely enhance after $\mathrm{Gd}$ injection. ${ }^{4} \mathrm{~A}$ triple dose of Gd-DTPA has proved to be safe and well tolerated ${ }^{8}$ and to increase greatly the diagnostic reliability of MRI for detection of enhancing lesions in several neurological diseases, including multiple sclerosis. ${ }^{8-12}$ There is at present no study which has tested the possible role of the triple dose of Gd-DTPA in patients with PPMS. As we now have treatment with beneficial effects on multiple sclerosis ${ }^{1314}$ and patients with PPMS are those with a highly disabling course, definition of markers of disease activity potentially useful for monitoring treatment is mandatory. This study had two aims: firstly to evaluate whether the triple dose of GdDTPA might increase the number of enhancing lesions in patients with PPMS and secondly to provide new information about the characteristics of the breakdown of the blood-brain barrier in this form of the disease.

\section{Patients and methods}

PATIENTS

Ten patients with primary progressive multiple sclerosis (mean age 40, range 26-57 years; mean duration of the disease $=6.5$, range $=2-11$ years; mean expanded disability status scale $^{15}$ score $4 \cdot 6$, range $3 \cdot 5-8 \cdot 0$ ) were selected from the multiple sclerosis population attending the Multiple Sclerosis Centre of the Scientific Institute Ospedale San Raffaele, University of Milan. Primary progressive multiple sclerosis was defined as a disease that was progressive from the onset, with no evidence of relapses or remissions. Written informed consent was obtained from all the patients before inclusion in the study.

MRI

Moderately T2 weighted MRI of the brain was performed with a 1.5 Tesla machine (SE $2000 / 50,5 \mathrm{~mm}$ contiguous axial slices, 256 $\times 256$ image matrix, field of view $220 \mathrm{~mm}$ ) at the same time that standard dose enhanced MRI was obtained. Lesion volumes present on $\mathrm{T} 2$ weighted scans were measured by one of us (MF) by a semiautomated thresholding 
technique. ${ }^{16} \mathrm{~T} 1$ weighted images (SE $768 / 14$, $5 \mathrm{~mm}$ contiguous axial slices, $256 \times 256$ image matrix, field of view $220 \mathrm{~mm}$ ) were obtained four times: five to seven minutes after the injection of the standard dose $(0 \cdot 1$ $\mathrm{mmol} / \mathrm{kg}$ ) of Gd-DTPA (Schering, Berlin, Germany), before, five to seven minutes, and 60 minutes after the injection of the triple dose $(0.3 \mathrm{mmol} / \mathrm{kg})$ of Gd-DTPA. Post-triple dose Gd-DTPA scans were obtained six to 24 hours after the standard dose scan was performed, when the T1 weighted scan performed before the injection of the triple dose of Gd-DTPA indicated that no enhancing lesions could be seen. Patients were repositioned according to published guidelines. ${ }^{3}$ Image review of enhanced T1 weighted scans in terms of the numbers of enhancing lesions was performed in three stages, with T2 weighted images as the gold standard:

\section{Stage 1}

Each of the three sets of T1 weighted images from each patient was reviewed independently by two of us (MF and AC), unaware of the Gd-DTPA dosage used and the patient to whom the scans belonged. Each lesion was scored according to size (small: diameter $\leqslant 5$ $\mathrm{mm}$; intermediate: diameter $6-10 \mathrm{~mm}$; large: diameter $>10 \mathrm{~mm}$ )

\section{Stage 2}

Next, the three sets of images were reviewed together and each lesion was reassessed.

\section{Stage 3}

Finally, the three sets of images were compared and each lesion once again reassessed.

The areas and the contrast ratios (CRs) for all the regions which were considered enhancing lesions at the end of this review were measured by a single rater (MF), using a mouse controlled cursor on the computer display. The CR was calculated by dividing the mean signal intensity of each lesion by the mean signal intensity (weighted for the number of pixels) obtained for two to six areas in the CSF. The areas in the CSF chosen were usually in the same slices in which the lesions were present. When this was not possible, the slices closest to the lesions in which CSF was present were considered.

\section{STATISTICAL ANALYSIS}

The differences in total enhancing area per patient (only for patients with at least one enhancing lesion) were studied by the Kruskall-Wallis test and post hoc analysis was performed with the Mann-Whitney test, as the data were not distributed normally. The differences in CR between enhancing lesions detected with the standard dose of GdDTPA, the triple dose of Gd-DTPA, and in the one hour delayed scans after the injection of the triple dose of Gd-DTPA were studied by one way analysis of variance (ANOVA). Post hoc analysis was performed with the two tailed Student's $t$ test for non-impaired data.
Results

The mean lesion load on T2 weighted images was $1970 \mathrm{~mm}^{2}$ (range $175-3790 \mathrm{~mm}^{2}$ ). Two patients showed four enhancing lesions when the standard dose of Gd-DTPA was given (one of the patients had one intermediate lesion and the other had one small and two intermediate lesions). No enhancing lesions were detected on the $\mathrm{T} 1$ weighted images obtained six to 24 hours after the standard dose scans before the injection of the triple dose of Gd-DTPA. After the injection of the triple dose of Gd-DTPA, three of the eight (41\%) patients with no enhancing lesions on the standard dose scans had three such lesions (two were small and one was intermediate; figure). In the patient who already had one enhancing lesion in the standard dose scan, we found seven enhancing lesions (five small and two intermediate) in the triple dose scan. In the other patient with three enhancing lesions in the standard dose scan, no change in number and size of enhancing lesions was found in the triple dose scan.

When the one hour delayed scan was performed after the injection of the triple dose of Gd-DTPA, another two enhancing lesions not present in the previous two scans were detected. One small lesion was found in a patient who had had no enhancing lesions in the other two scans. A second intermediate lesion was found in another patient who had had one enhancing lesion in the early triple dose scan. In addition, in the patient with seven enhancing lesions in the triple scans, one of the small lesions had become intermediate in the one hour delayed scan, and one small lesion was no longer detectable in the patient who had three enhancing lesions in the standard dose and triple dose scans. In the same patient, one intermediate lesion had become small.

In summary, four enhancing lesions were detected in the standard dose scans, 13 in the triple dose scans, and 14 in the one hour delayed scan. All these lesions enhanced homogeneously at each dose and were present on the T2 weighted scans. The number of "active" scans increased from two with the standard dose of Gd-DTPA, to six in both the triple dose scans.

The mean enhancing area/patient was 6.8 $\mathrm{mm}^{2}$ (range $0-25.6 \mathrm{~mm}^{2}$ ) with the standard dose of Gd-DTPA, $28.6 \mathrm{~mm}^{2}$ (range 0-136.4 $\mathrm{mm}^{2}$ ) with the triple dose, and $38.4 \mathrm{~mm}^{2}$ (range 4-175.1 $\mathrm{mm}^{2}$ ) in the one hour delayed scans. None of these differences were significant, perhaps because of the small size of the sample.

The mean CR (SD) was $2.6(0.1)$ for the lesions detected in the standard dose scans, $3.7(0.5)$ for the lesions detected in the triple dose scans, and $3.2(0.8)$ for those in the one hour delayed scans $(P=0.009)$. The $C R$ for enhancing lesions detected with the triple dose of Gd-DTPA was higher than those for enhancing lesions present in both the standard dose scans $(P<0.0009)$ and the one hour delayed scans $(P=0.04)$.

No patient complained of discomfort or 
(A) Moderate T2 weighted (SE 2000/50) axial image. Two hyperintense lesions are visible in the right part of the floor of the fourth ventricle and in the right cerebellar hemisphere. (B) $T 1$ weighted ( $S E$ 768/14) axial image obtained five to seven minutes after the injection of the standard dose $(0.1 \mathrm{mmol} / \mathrm{kg})$ of gadolinium-DTPA. None of these lesions are enhanced. (C) $T 1$ weighted (SE 768/14) axial image obtained five to seven minutes after the injection of the triple dose $(0.3 \mathrm{mmol} / \mathrm{kg})$ of gadolinium-DTPA. The lesion in the right part of the floor of the fourth ventricle is enhanced. (D) $T 1$ weighted (SE 768/14) axial image obtained one hour after the injection of the triple dose $(0.3 \mathrm{mmol} / \mathrm{kg})$ of gadolinium-DTPA. The lesion in the right part of the floor of the fourth ventricle is still enhancing, and its diameter is larger.

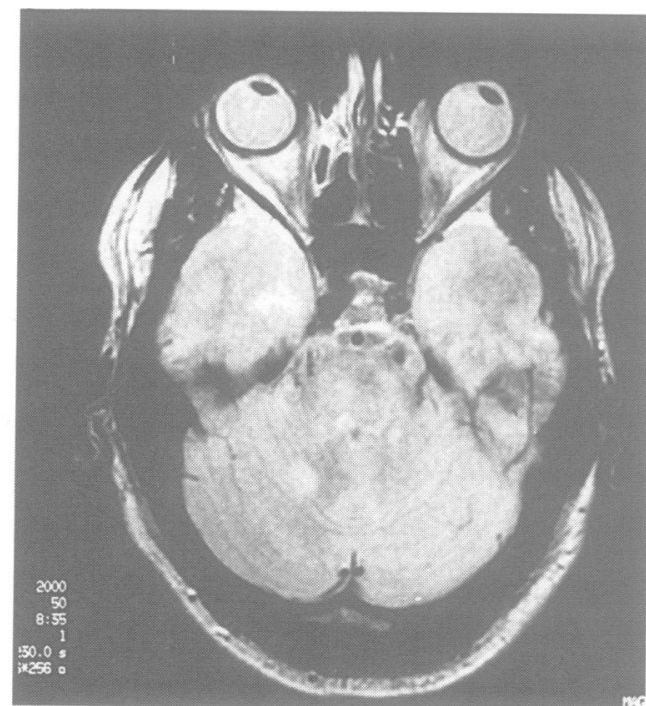

(A)

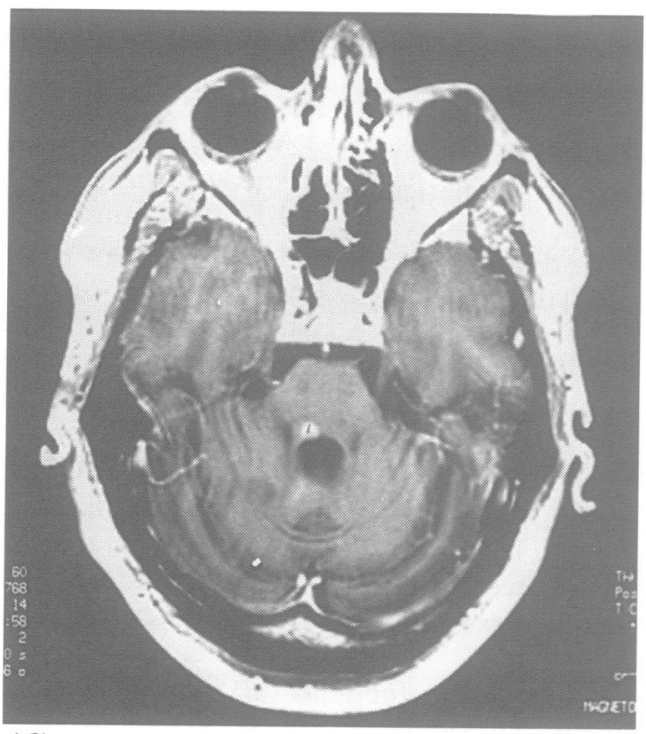

(C)

presented side effects after the injection of the triple dose of Gd-DTPA.

\section{Discussion}

Previous studies showed that giving a triple dose of contrast material improves detection of lesions in several neurological diseases, including multiple sclerosis. ${ }^{8-12}$ There are no studies, however, evaluating the role of the triple dose of Gd-DTPA in the different clinical subgroups of multiple sclerosis. It is well known that the number, extent, and frequency of enhancing lesions vary greatly in the different clinical subgroups of the disease $^{2-6} 17$ and that patients with PPMS are those in whom enhancing lesions are hard to detect. Thompson $e t a l^{4}$ found only one enhancing lesion in the brains of 12 patients with PPMS followed up for six months with monthly MRI. Similar results have been more recently reported by Kidd et al ${ }^{18}$ who studied 10 patients with PPMS with both brain and spinal cord MRI monthly. The results of the present study indicate that using the triple dose of Gd-DTPA in such patients enables us to detect more enhancing lesions than when
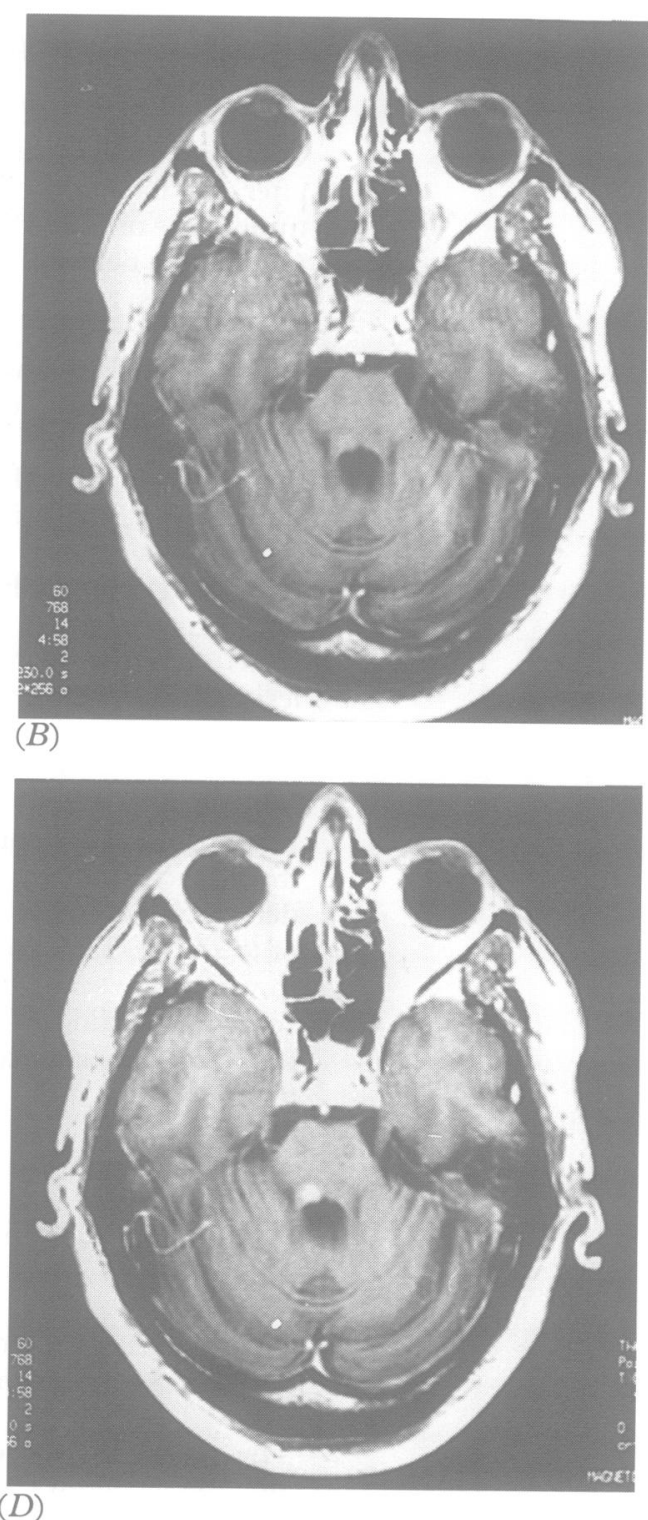

using the standard dose. With the triple dose of Gd-DTPA, both the number of enhancing lesions and the number of "active" scans increased by about $300 \%$. The sizes of the enhancing lesions were also larger and their CR better than those obtained with the standard dose.

These results imply, firstly, that inflammation is not an unusual finding in patients with PPMS. Enhancement by Gd is secondary to increased permeability of the blood-brain barrier, which is associated with inflammation both in chronic relapsing experimental allergic encephalomyelitis ${ }^{1920}$ and in multiple sclerosis. $^{21}$ By contrast, non-inflammatory demyelination is not accompanied by changes in permeability of the blood-brain barrier. ${ }^{22}$ The presence of enhancing lesions in multiple sclerosis is, therefore, considered to reflect the presence of active inflammation. A previous pathological study ${ }^{23}$ showed that there is indeed inflammation in the lesions of patients with PPMS and that it was just less intense than that detectable in patients with secondary progressive multiple sclerosis. Our in vivo data confirm this finding. We found more enhancing lesions when the triple dose 
of Gd-DTPA was used, but the total number of such lesions was only $13(1.3$ enhancing lesions per patient) against the 127 enhancing lesions we have detected in 15 patients with either relapsing-remitting or secondary progressive multiple sclerosis $(8.5$ enhancing lesions per patient) using the same study protocol (unpublished data).

Previous studies showed that delayed high dose CT is useful for displaying the vasoactive lesions of multiple sclerosis. $^{24-26}$ Nevertheless, at present there are no studies evaluating the possible role of delayed MRI in patients with PPMS. In these patients, in whom the inflammatory process seems to be less intense, it is possible that a longer time is needed for the contrast material to leak into "active" lesions. Our data indicate that it is worthwhile in patients with PPMS to obtain a second delayed scan after the triple dose GdDTPA injection, as we detected two more enhancing lesions which were not seen in the first triple dose scan and a patient considered to be "inactive" was moved to the category of "active" patients.

These data suggest that enhancing lesions in patients with PPMS might be heterogeneous in their degree of inflammation. There is a small group of lesions which enhance with the standard dose of GdDTPA. These lesions are probably characterised by an inflammatory process with an intensity not very different from that present in patients with relapsing-remitting and secondary progressive multiple sclerosis. A second, larger, group of lesions enhance only when a higher transmembrane concentration of contrast material has been reached. These lesions are probably those in which inflammation and permeability of the blood-brain barrier are less intense, as already shown pathologically. ${ }^{23}$ In the final group of lesions enhancement can be seen only when both the triple dose and delayed scanning are used. It may be argued that in these lesions the permeability of the blood-brain barrier is extremely modest and longer periods of time are needed for the contrast material to reach a concentration within the lesions high enough to be visible.

The second implication, which arises as a consequence of previous considerations, is that the use of a triple dose of Gd-DTPA might render patients with PPMS suitable for phase II clinical trials in which changes in the number and extent of enhancing lesions are the most commonly used end points. ${ }^{7}$ This is particularly true as we found four patients who would be considered as "non-active" if only the standard dose of Gd-DTPA had been used, moved into the category of "at least one enhancing lesion", when the triple dose was used. Two things must be considered, however. Firstly, the numbers and the extent of enhancing lesions detectable with the triple dose are still low and large samples of patients will need to be enrolled in clinical trials using such end points. This limitation might be, at least partially, overcome by the combined use of high dose contrast material and magnetisation transfer contrast, which has proved to be more sensitive than high dose contrast material alone in increasing detectability of enhancing lesions. ${ }^{27}$ Secondly, longitudinal studies are needed to calculate the rate of development of enhancing lesions with the triple dose of Gd-DTPA. In this respect, the main limitation, which applies also to clinical trials, is that the safety of monthly use of the triple dose of Gd-DTPA in longitudinal studies, lasting for at least six to 12 months, has not been established.

The third implication is that a triple dose of Gd-DTPA might be useful for diagnostic purposes for patients suspected to have PPMS. Because white matter lesions, which are often small and punctate, are often seen in healthy adults over the age of $50^{28} 29$ and the onset of PPMS is typically late, the finding of areas of enhancement could aid in establishing the diagnosis. The same consideration is valid when the cause of a progressive spastic paraparesis has to be found, as lesions on T2 weighted scans are not unusual in other neurological conditions. ${ }^{30-33}$

In conclusion, our study indicates that the triple dose of Gd-DTPA is of importance in the clinical evaluation of patients with PPMS, as it is useful for increasing the diagnostic certainty and for defining patients in an active phase of the disease. It also indicates that obtaining a delayed scan after the injection of the triple dose further increases the possibility of detecting enhancing lesions.

The program for lesion area measurements was written by Drs D A G Wicks, P S Tofts, G J Barker, and M A Horsfield in the NMR Research Group, Institute of Neurology, Queen Square, London, UK, with generous financial assistance from the Multiple Sclerosis Society of Great Britain and Northern Ireland. The Dispimage package display program was written by $\mathrm{Mr} \mathrm{D}$ Plummer, Department of Medical Physics, University College London, UK. We are greatly indebted to Dr M A Horsfield, Department of Medical Physics, Roya Leicester Infirmary, Leicester, UK for his help in the loca implementation of the software and to $\mathrm{Mr} \mathrm{C}$ Pereira for his skillful technical assistance.

1 Kermode AG, Tofts PS, Thompson AJ, et al. Heterogeneity of blood-brain barrier changes in multiple sclerosis: an MRI study with gadoliniumDTPA enhancement. Neurology 1990;40:229-35.

2 Harris JO, Frank JA, Patronas N, McFarlin DE, McFarland HF. Serial gadolinium-enhanced magnetic resonance imaging scans in patients with early, relapsing-remitting multiple sclerosis: implications for clinical trials and natural history. Ann Neurol 1991;29:548-55.
Miller DH, Barkhof F, Berry I, Kappos L, Scotti G, Miller DH, Barkhof F, Berry I, Kappos L, Scotti G,
Thompson AJ. Magnetic resonance imaging in monitoring the treatment of multiple sclerosis: concerted action guidelines. I Neurol Neurosurg Psychiatry 1991;54:683-8.

4 Thompson AJ, Kermode AG, Wicks D, et al. Major differences in the dynamics of primary and secondary progressive multiple sclerosis. Ann Neurol 1991;29:53-62.

5 Smith ME, Stone LA, Albert PS, et al. Clinical worsening in multiple sclerosis is associated with increased frequency and area of gadopentetate dimeglumineenhancing magnetic resonance imaging lesions. Neurol 1993;33:480-9.

6 Kidd D, Thompson AI, Kendall BE Miller McDonald WI Ben AJ, Kendall BE, Miller DH, McDonald WI. Benign form of multiple scleroris: MR evidence for less frequent and less inflammatory diseas

7 Miller DH. Magnetic resonance in monitoring the treatment of multiple sclerosis. Ann Neurol 1994;36:S91-4.

8 Haustein J, Laniado M, Niendorf HP, et al. Triple-dose versus standard-dose gadopentetate dimeglumine: randomised study of 199 patients. Radiology 1993;186: 855-60.

9 Yuh WTC, Fisher DJ, Mayr-Yuh NA, et al. Review of the use of high-dose gadoteridol in the magnetic resonance evaluation of central nervous system tumors. Invest Radiol 1992;27:539-44. 
10 Runge VM, Kirsch JE, Burke VI, et al. High-dose gadoteridol in MR imaging of intracranial neoplasms. $f$ Magn Reson Imaging 1992;2:9-18.

11 Runge VM, Kirsch JE, Thomas GS. High-dose applications of gadolinium chelates in magnetic resonance ima

12 Wolansky LJ, Bardini JA, Cook SD, Zimmer AE, Sheffet A, Lee HJ. Triple-dose versus single-dose gadoteridol in multiple sclerosis patients. Fournal of Neuroimaging multiple scler

13 The IFNB Multiple Sclerosis Study Group. Interferonbeta-1b is effective in relapsing-remitting MS.I. Clinical results of a multicenter, randomized, double-blind, placebo-controlled trial. Neurology 1993;43:655-61.

14 Paty DW, Li DBK, the UCB MS/MRI Study Group IFNB Multiple Sclerosis Study Group. Interferon beta$1 \mathrm{~b}$ is effective in relapsing-remitting multiple sclerosis. II. MRI analysis results of a multicenter, randomized, double-blind, placebo-controlled trial. Neurology 1993; 43:662-7.

15 Kurtzke JF. Rating neurologic impairment in multiple sclerosis: an expanded disability status scale (EDSS). Neurology 1983;33:1444-52.

16 Wicks DAG, Tofts PS, Miller DH, et al. Volume measurements of multiple sclerosis lesions with magnetic surements of multiple sclerosis lesions with magnetic 1992;34:475-9.

17 Filippi M, Campi A, Mammi S, et al. Brain magnetic resonance imaging and multimodal evoked potentials in benign and secondary progressive multiple sclerosis. f Neurol Neurosurg Psychiatry 1995;58:31-7.

18 Kidd D, Kendall BE, Barker GJ, et al. A monthly seria study of the brain and spinal cord in progressive multiple sclerosis [abstract]. Proceedings of the Society of Magnetic Resonance 1994;1:165.

19 Hawkins CP, Munro PMG, MacKenzie F, et al. Duration and selectivity of blood-brain barrier breakdown in chronic relapsing experimental allergic encephalomyelitis studied by padolinium-DTPA and protein markers. Brain 1990;113:365-78.

20 Hawkins CP, MacKenzie F, Tofts P, duBoulay EPGH McDonald WI. Patterns of blood-brain barrier breakdown in inflammatory demyelination. Brain 1991;114: 801-10.

21 Katz D, Taubenberger JK, Cannella B, McFarlin DE, Raine CS, McFarland HF. Correlation between mag netic resonance imaging findings and lesion develop- ment in chronic, active multiple sclerosis. Ann Neurol 1993;34:661-9.

22 Lumdsen CE. The neuropathology of multiple sclerosis In: Vinken PJ, Bruyn GW, eds. Handbook of clinical neurology. Vol 9. Amsterdam: North Holland, 1970: 217-309.

23 Reverz T, Kidd D, Thompson AJ, Barnard RO, McDonald WI. A comparison of pathology of primary and secondary progressive multiple sclerosis. Brain 1994;117:759-65.

24 Vinuela FV, Fox AI, Debrun GM, Feasby TE, Ebers GC. New perspectives in computed tomography of multiple New perspectives in computed tomography of m
sclerosis. AfNR Am $₹$ Neuroradiol 1982;3:277-81.

25 Sears S, McCammon A, Bigelow R, Hayman LA. Maximizing the harvest of contrast enhancing lesions in multiple sclerosis. Neurology 1982;32:815-20.

26 Spiegel SM, Vinuela F, Fox AJ, Pelz DM. CT of multiple sclerosis: reassessment of delayed scanning with high doses of contrast material. AfR Am $\mathcal{f}$ Roentgenol 1985; 145:497-500.

27 Finelli DA, Hurst GC, Gullapali RP, Bellon EM. Improved contrast of enhancing brain lesions on postgadolinium T1 weighted spin-echo images with use of magnetization transfer. Radiology 1994;36:62-7.

28 Gerard G, Weisberg LA. MRI periventricular lesions in adults. Neurology 1986;36:998-1001.

29 Awad IA, Johnson PC, Spetzler RF, Hodak JA. Incidental subcortical lesions identified on magnetic resonance imaging in the elderly. II. Postmortem pathological imaging in the elderly. II. Postm

30 Kinkel WR, Jacobs L, Polachini I, Bates V, Heffner RR. Subcortical atherosclerotic encephalopathy (Binswanger's disease). Computed tomographic, nuclear magnetic resonance and clinical correlations. Arch Neurol 1985;42:951-9.

31 Miller DH, Ormerod IEC, Gibson A, duBoulay EPGH, Rudge P, McDonald WI. MR brain scanning in patients with vasculitis: differentiation from multiple sclerosis. Neuroradiology 1987;29:226-31.

32 Waldemar G, Christiansen P, Larsson HWB, et al. White matter hyperintensities in dementia of Alzheimer type: morphological and regional cerebral blood flow correlates. $₹$ Neurol Neurosurg Psychiatry 1994;57:1458-65.

33 Harding AE, Sweeney MG, Miller DH, et al. Occurrence of a multiple sclerosis-like illness in women who have a of a multiple sclerosis-like ilness in women who have a Leber's hereditary optic neuropathy
DNA mutation. Brain 1992;115:979-89. 